\title{
A perverse quality incentive in surgery: implications of reimbursing surgeons less for doing laparoscopic surgery
}

\author{
Amanda N. Fader ${ }^{1}$ Tim Xu X Brian J. Dunkin $^{4,5} \cdot$ Martin A. Makary ${ }^{2,3,6}$
}

Received: 6 July 2016/Accepted: 5 August 2016/Published online: 17 October 2016

(C) Springer Science+Business Media New York 2016

\begin{abstract}
Background Surgery is one of the highest priced services in health care, and complications from surgery can be serious and costly. Recently, advances in surgical techniques have allowed surgeons to perform many common operations using minimally invasive methods that result in fewer complications. Despite this, the rates of open surgery remain high across multiple surgical disciplines.

Methods This is an expert commentary and review of the contemporary literature regarding minimally invasive surgery practices nationwide, the benefits of less invasive approaches, and how minimally invasive compared with open procedures are differentially reimbursed in the United States. We explore the incentive of the current surgeon reimbursement fee schedule and its potential implications. Results A surgeon's preference to perform minimally invasive compared with open surgery remains highly
\end{abstract}

Martin A. Makary

mmakary1@jhmi.edu

1 Kelly Gynecologic Oncology Service, Department of Gynecology and Obstetrics, Johns Hopkins Medicine, Baltimore, MD, USA

2 Department of Surgery, Johns Hopkins Medicine, Baltimore, MD, USA

3 Department of Surgery, Weill Cornell Medical College, New York City, NY, USA

4 Department of Surgery, Houston Methodist Hospital, Houston, TX, USA

5 Department of Health Policy and Management, Johns Hopkins University School of Medicine, Baltimore, MD, USA

6 Surgery and Health Policy and Management, Johns Hopkins University School of Medicine, Halsted 610, 600 N. Wolfe St, Baltimore, MD 21287, USA variable in the U.S., even after adjustment for patient comorbidities and surgical complexity. Nationwide administrative claims data across several surgical disciplines demonstrates that minimally invasive surgery utilization in place of open surgery is associated with reduced adverse events and cost savings. Reducing surgical complications by increasing adoption of minimally invasive operations has significant cost implications for health care. However, current U.S. payment structures may perversely incentivize open surgery and financially reward physicians who do not necessarily embrace newer or best minimally invasive surgery practices.

Conclusions Utilization of minimally invasive surgery varies considerably in the U.S., representing one of the greatest disparities in health care. Existing physician payment models must translate the growing body of research in surgical care into physician-level rewards for quality, including choice of operation. Promoting safe surgery should be an important component of a strong, value-based healthcare system. Resolving the potentially perverse incentives in paying for surgical approaches may help address disparities in surgical care, reduce the prevalent problem of variation, and help contain health care costs.

Keywords Minimally invasive surgery $\cdot$ Reimbursement · Incentives · Quality

Surgery is one of the highest priced services in health care, and complications from surgery can be serious and costly. Recently, advances in surgical techniques have matured to allow surgeons to perform many common operations using minimally invasive methods that result in fewer complications. Yet surgeon preference to perform the minimally invasive operation, versus open surgery, even after 
Table 1 Physician payments and RVUs for common operations with a minimally invasive approach option

\begin{tabular}{llc}
\hline Surgery & \multicolumn{2}{l}{ Physician payment (professional fee) } \\
\cline { 2 - 3 } & Open & Minimally invasive \\
\hline Hysterectomy & $\$ 1040(28.9)$ & $\$ 839(23.4) / \$ 729(20.3)^{*}$ \\
Cholecystectomy & $\$ 1110(31.0)$ & $\$ 683(19.1)$ \\
Colectomy, total, with ileocolostomy & $\$ 1947(54.3)$ & $\$ 1854(51.7)$ \\
Lung lobectomy & $\$ 1541(43.0)$ & $\$ 1461(40.8)$ \\
\hline
\end{tabular}

* Vaginal and laparoscopic approaches; for a uterus $250 \mathrm{~g}$ or less adjusting for differences in patient complexity, remains highly variable [1]. This represents one of the greatest disparities in health care today. In this article, we explore the incentive of the current surgeon reimbursement fee schedule and its potential implications for patient complications and cost in the USA.

Hysterectomy is the second most common operation performed in the USA and can often be performed by laparoscopy for both benign and malignant gynecologic conditions. Despite Level 1 evidence and USA. Nationwide Inpatient Sample data [1, 2] affirming what many consider to be common sense-that minimally invasive hysterectomy has fewer complications compared to open surgery, even in obese or comorbid patients-the Centers for Medicare and Medicaid Services (CMS) pays surgeons more to perform the more morbid, open operation (Table 1). The difference in reimbursement is even greater for women with uteri weighing $<250 \mathrm{~g}$, the subgroup most likely to benefit from laparoscopy. This modified payment incentive can be a barrier to a more appropriate procedure choice for patients who are candidates for minimally invasive surgery, financially rewarding physicians who do not learn, offer, or perform this approach. The hysterectomy example is one of the most vivid, but payments for other common operations also need reform to help advance surgical quality and safety.

One of the greatest challenges is to deliver high-quality care to patients while controlling costs remains varying in clinical practice despite clear evidence to support standardization and best practices in several disciplines. Barriers to the implementation of evidence-based care include lack of awareness regarding evidence, uneducated disagreement with the evidence, and clinical inertia (awareness and agreement about the evidence, but lack of adoption) [3]. In the case of minimally invasive surgery, there is also a time-consuming learning curve to acquire modern technical skills. If a surgeon did not learn how to do a procedure minimally invasively during their training, it can be quite challenging to learn it once in practice. The procedures are quite different from open surgery, and there is no national infrastructure for training practicing surgeons to do new procedures, limited ability to get hands-on experience during training, lack of available support for mentorship in the OR while adopting the new procedure, and difficulty in taking time away from a busy practice including loss of service to patients and loss of income. These barriers, coupled with lower payment for the minimally invasive procedures, slow adoption. In fact, it may take a generation or two for best practices to disseminate widely.

Keeping surgeons' technical skills up to date has been a vexing challenge for professional associations and accrediting boards. While surgeons are required to regularly participate in continuing medical education and take written examinations for board re-certification, these efforts focus entirely on knowledge with technical skills entirely absent from this process. In-person coaching programs have recently been described as valuable to surgeons who wish to learn new technical skills [4], and tele-mentoring into the operating room using audio-video technology has also been used to support surgeons through the early part of their learning curve. Unfortunately, these forms of instruction are vastly underutilized.

While many surgeons do their best to learn and master state-of-the-art operations, others follow incentives to do otherwise, sticking with riskier operation choices for their patients, such as open surgery, even when patients are minimally invasive surgery candidates. As US hospital systems merge and expand, surgeons may be incentivized to adopt best practice, and the use of surgical coaching and video tele-mentoring could aid in this process. These tools may help healthcare networks achieve the Institute of Medicine's goal of creating a learning healthcare system [5].

Many physicians do not respond at all to perverse financial incentives, but studies suggest that some do. One study of urologists found that when they own radiation therapy equipment, they are more likely to prescribe radiation treatment [6]. On the other hand, hospital pay-forperformance schemes intended to incentivize quality have had limited results [7]. This may be because the incentive is too small or too diffuse (e.g., paid to a large organization rather than directly to the physician), or that a retooling infrastructure is not available to foster adoption of best 
practice. By contrast, the current procedure-specific reimbursement schedule for the physician, separate from the hospital payment, contains strong reverse incentives.

The context for current physician payments lies in the history of the relative value unit (RVU) system. Since 1992, a committee of physicians has decided on the number of RVUs to allocate to each medical procedure or service, depending on the physician's time, mental effort, and technical skill required to manage the disease. The Center for Medicare and Medicaid Services (CMS) and private insurers then convert these RVUs proportionally to physician fee reimbursements. While not all minimally invasive operations have fewer RVUs compared to open operations, we highlight common operations where the payment model does not reward surgeons adopting best practices for candidate patients (Table 1). RVUs were created to reimburse labor; they were not developed with the intention of rewarding best practices. As a result, our healthcare payment system must be reformed to reimburse surgeons based on patient complexity and use of best practices. Minimally invasive surgery can be technically challenging and may require more advanced skills. At minimum, surgeons should not be paid less for performing the safer operation.

Reducing surgical complications by increasing adoption of minimally invasive operations also has significant cost implications for health care. Using nationwide administrative claims data, we previously reported that the use of minimally invasive surgery in place of open surgery is associated with reduced adverse events and hospital length of stay, accompanied by an estimated cost savings of $\$ 1500$ per appendectomy, $\$ 7500$ per colectomy, and $\$ 6300$ per lung lobectomy. Our research indicates that for these three common procedures, increasing minimally invasive surgery utilization by $50 \%$ would save over $\$ 300$ million annually across the US healthcare system [8].

Currently, payment structures incentivize the wrong operation and financially reward doctors who do not embrace newer best practices. While many physicians do not respond to perverse financial incentives, payment models must translate the growing body of research in surgical care into physician-level rewards for quality, including choice of operation. Given the prevalence and cost of surgery, promoting safe surgery should be an important prerequisite for creating a stronger, value-based healthcare system. Resolving the potentially perverse incentives in paying for surgical approaches may help address disparities in surgical care, reduce the endemic problem of variation, and help constrain healthcare inflation in the USA.

Funding Funded by a gift from the Mr. and Mrs. Rodda Family Foundation.

\section{Compliance with ethical standards}

Disclosures Amanda N. Fader, Tim Xu, Brian J. Dunkin, and Martin A. Makary have no conflicts of interest or financial ties to disclose.

\section{References}

1. Cooper MA, Hutfless S, Segev DL et al (2014) Hospital level under-utilization of minimally invasive surgery in the United States: retrospective review. BMJ 349:g4198

2. Fader AN, Weise RM, Sinno AK et al (2016) Utilization of minimally invasive surgery in endometrial cancer care: a quality and cost disparity. Obstet Gynecol 127:91-100

3. Cabana MD, Rand CS, Powe NR et al (1999) Why don't physicians follow clinical practice guidelines? A framework for improvement. JAMA 282:1458-1465

4. Hu YY, Peyre SE, Arriaga AF et al (2012) Postgame analysis: using video-based coaching for continuous professional development. J Am Coll Surg 214:115-124

5. Mitchell JM (2013) Urologists' use of intensity-modulated radiation therapy for prostate cancer. N Engl J Med 369:1629-1637

6. Olsen L, Aisner D, and McGinnis JM (eds) (2007) The learning healthcare system: workshop summary. Institute of Medicine Roundtable on Evidence-Based Medicine, National Academies Press (US)

7. Jha AK (2013) Time to get serious about pay for performance. JAMA 309:347-348

8. Xu T, Hutfless SM, Cooper MA, Zhou M, Massie AB, Makary MA (2015) Hospital cost implications of increased use of minimally invasive surgery. JAMA Surg 150:489-490 